1 Centro Universitário Uninovafapi - Teresina (PI), Brasil.

m.dnunes@hotmail.com

2 Universidade Estadual do Piauí (Uespi) - Teresina

(PI), Brasil.

3 Universidade de Brasília (UnB) - Brasília (DF),

Brasil.

\section{Mortes maternas por aborto entre adolescentes no Piauí, Brasil}

\section{Maternal deaths due to abortion among adolescents in Piauí, Brazil}

Maria das Dores Sousa Nunes ${ }^{\mathbf{1}}$, Alberto Madeiro², Debora Diniz ${ }^{\mathbf{2}}$

DOI: $10.1590 / 0103-1104201912312$

RESUMO Este artigo analisa as mortes maternas de adolescentes no Piauí e descreve as histórias daquelas que morreram por aborto induzido entre 2008 e 2013. O estudo foi realizado em duas etapas. A primeira, quantitativa, obteve dados demográficos e causas básicas dos óbitos do Sistema de Informações de Mortalidade. Na segunda, qualitativa, foram entrevistadas as mães das adolescentes. As mortes de adolescentes representaram 17,2\% (50 casos) do total de óbitos maternos. A maior parte das jovens residia em cidades do interior (78\%) e era negra (70\%). Destacaram-se como causas dos óbitos transtornos hipertensivos (28\%), infecção puerperal (16\%), hemorragia (12\%), tromboembolismo (12\%) e aborto (10\%). O uso de medicamento ocorreu em todos os casos de aborto, sendo sangramento abundante e dor pélvica os principais motivos para a busca de atendimento hospitalar. Houve demora no diagnóstico e no tratamento adequado das complicações do aborto, o que pode ter contribuído para a morte das adolescentes. As mortes maternas de adolescentes foram causadas por condições consideradas evitáveis. As histórias das jovens que morreram por complicações do aborto evidenciaram a necessidade de assistência em saúde rápida e disponível, além de leis e políticas públicas que protejam as mulheres que decidem interromper a gravidez.

PALAVRAS-CHAVE Mortalidade materna. Aborto. Adolescente.

\begin{abstract}
This article analyzes maternal deaths of adolescents in Piaui and describes the stories of those who died due to induced abortion between 2008 and 2013. The study was conducted in two stages. The first, quantitative, obtained demographic data and basic causes of deaths from the Mortality Information System. In the second, qualitative, the mothers of the adolescents were interviewed. Adolescents' deaths accounted for $17.2 \%$ (50 cases) of total maternal deaths. The majority of the adolescents lived in inner cities (78\%) and was black (70\%). The causes of death were hypertensive disorders (28\%), puerperal infection (16\%), hemorrhage (12\%), thromboembolism (12\%) and abortion (10\%). The use of medication occurred in all cases of abortion, with abundant bleeding and pelvic pain being the main reasons for seeking hospital care. There was delay in the diagnosis and appropriate treatment of abortion complications, which may have contributed to the death of the adolescents. Maternal deaths among adolescents were mostly caused by conditions considered preventable. The stories of young women who died of abortion complications have highlighted the need for better-qualified health care, as well as laws and public policies that protect women who decide to terminate their pregnancies.
\end{abstract}

KEYWORDS Maternal mortality. Abortion. Adolescent. 


\section{Introdução}

A gravidez na adolescência é evento de importante magnitude e marcador de vulnerabilidade social. Embora tenha havido redução das taxas de gravidez nas últimas duas décadas, cerca de 16 milhões de adolescentes entre 15-19 anos e 2,5 milhões daquelas abaixo de 16 anos dão à luz em países em desenvolvimento ${ }^{1}$. No Brasil, houve decréscimo de $17 \%$ no número de adolescentes grávidas entre 2003 e 2015, porém, 1 a cada 5 partos ainda é de jovens com 19 anos ou menos ${ }^{\mathbf{2}, \mathbf{3}}$. As proporções mais elevadas são encontradas entre as adolescentes de baixa escolaridade, negras e residentes na zona rural das regiões Norte e Nordeste do País ${ }^{4-6}$.

Com gestações frequentemente não planejadas, o aborto é uma possibilidade para muitas adolescentes, realizado de forma insegura em grande parte dos casos, ${ }^{7,8}$. Em todo o mundo, embora tenha sido observada diminuição das taxas de interrupção da gravidez entre 1990 e 2008, a cada ano, cerca de 4,5 milhões de adolescentes ainda realizam aborto, com taxas mais elevadas nos países em desenvolvimento ${ }^{9,10}$. Não existem números específicos sobre aborto entre adolescentes no Brasil, porém, a Pesquisa Nacional do Aborto, que, em 2016, entrevistou 2.002 mulheres urbanas e alfabetizadas, verificou que $9 \%$ daquelas com idade entre 18 e 19 anos relataram já ter feito um aborto. Além disso, foi observado que houve maior frequência do último aborto entre mulheres mais jovens, com $29 \%$ deles ocorrendo entre 12 a 19 anos".

Uma revisão sistemática publicada em 2018 com dados de 28 países evidenciou que $23 \%$ dos casos hospitalizados de aborto em todas as idades evoluem para morbidade materna grave/ near miss, e 1,5\% deles para morte. Hemorragia foi a complicação relatada mais comum, porém, infecção se mostrou a mais frequente razão do óbito ${ }^{12}$. O atraso do sistema de saúde em garantir tratamento adequado para essas complicações, comumente determinado pelo estigma relacionado ao aborto, aumenta a chance de evolução para a morte ${ }^{13}$. Os dados brasileiros demonstram que o aborto está entre as cinco principais causas de morte materna, sendo responsável por 5 a $20 \%$ dos óbitos, contudo, é possível que a ilegalidade da prática no País possibilite a subnotificação dos casos ${ }^{14,15}$.

As principais causas de mortalidade materna entre adolescentes são similares às de mulheres mais velhas, com ênfase para as desordens hipertensivas, hemorragias e aborto ${ }^{16}$. No entanto, quando comparadas às mulheres de 20-24 anos, adolescentes tendem a apresentar complicações mais graves durante a gravidez e o parto ${ }^{17}$, sendo essa a causa mais comum de óbito entre mulheres de 15 a 19 anos no mundo ${ }^{13}$. Estudos brasileiros sobre morte entre adolescentes são mais raros do que em outras faixas etárias. Um obstáculo é o fato de que familiares se recusam a admitir que o aborto foi a causa do óbito da adolescente, seja pelo receio dos problemas legais e pelo estigma ou, ainda, por fragilidades no sistema de notificação da morte materna ${ }^{13,17}$. Há poucos dados que caracterizem os métodos, as práticas ou os itinerários que as adolescentes percorreram diante de uma gravidez, aborto, suas complicações e óbito, principalmente em regiões de grande vulnerabilidade socioeconômica e com menor oferta de serviços de saúde, como o Nordeste do Brasil. Este estudo teve o objetivo de analisar as mortes maternas de adolescentes no estado do Piauí, ocorridas entre 2008 e 2013, e conhecer as histórias daquelas que faleceram por complicações do aborto inseguro.

\section{Metodologia}

Trata-se de estudo transversal, realizado em duas etapas. Na primeira, de caráter quantitativo, houve levantamento das mortes maternas no Piauí, no período de janeiro de 2008 a dezembro de 2013, com ênfase nas mortes entre adolescentes. A segunda etapa, de natureza qualitativa, constituiu-se da análise das mortes de adolescentes por aborto. Os dados foram oriundos do Sistema de Informações de Mortalidade (SIM), do Sistema de Informação 
sobre Nascidos Vivos (Sinasc), cujo acesso ocorreu pelo Serviço de Vigilância do Óbito Materno do Piauí e de Teresina. Além disso, foram realizadas entrevistas com familiare ( $n=7)$ e amiga $(n=1)$ das adolescentes falecidas por aborto.

As variáveis da primeira etapa, derivadas da Declaração de Óbito (DO) e da ficha-síntese de investigação, foram as seguintes: faixa etária (em anos: 10 a 13, 14 a 17, 18 e 19); escolaridade (anos completos de estudo: nenhum, 1 a 3, 4 a 7, 8 a 12, mais de 12 anos, ignorada); raça/ cor da pele (branca, negra, outra, ignorada); estado civil (solteira, casada, viúva, separada, outra, ignorada); cidade de ocorrência (capital, interior); cidade de residência (capital, interior); número de gestações anteriores (nenhuma, 1, 2 ou mais, ignorado); realização de pré-natal (sim, não, ignorado); número de consultas pré-natais (nenhuma, 1 a 5, 6 ou mais, ignorado); tipo de resolução da gravidez (parto vaginal, parto cesáreo, não se aplica, ignorado); momento do óbito (durante a gravidez ou parto, após o parto, após o aborto); e causa básica do óbito (um dos códigos da Classificação Internacional de Doenças, versão 10 - CID10).

Para a segunda etapa, foram realizadas entrevistas com familiares ou amigas das adolescentes mortas por aborto. A mãe foi a participante que esteve presente em todas as entrevistas, mas irmãs, sogra e amigas também colaboraram. Todas as entrevistas foram gravadas, tendo o contato com os familiares das adolescentes sido intermediado por agentes de saúde locais. O local escolhido pelas participantes para a entrevista foi a própria residência. Um roteiro semiestruturado serviu para guiar as entrevistas, com questões centrais que recuperassem informações que a adolescente teria compartilhado com familiares ou amigas. As perguntas referentes ao aborto tentaram identificar o método utilizado (como medicamentos, chás ou ervas e/ou instrumentos introduzidos no útero) e a assistência em saúde recebida. As entrevistas buscaram as histórias não registradas nos documentos biomédicos oficiais, como, por exemplo, a descrição das decisões tomadas e os fatos ocorridos nas trajetórias das adolescentes até $\mathrm{o}$ adoecimento.
Os dados foram analisados descritivamente, com destaque para a inserção social e familiar da adolescente e as causas das mortes. Calculouse a razão de mortalidade materna (número de mortes maternas dividido pelo número de nascidos vivos, multiplicado por 100.000) para o período. As entrevistas foram examinadas pela técnica de análise de conteúdo, com definição de categorias sobre a prática de aborto, a assistência médica e as razões que culminaram na morte. Dessa forma, uma história circunstanciada de cada adolescente foi estruturada por informações oriundas tanto das entrevistas como dos dados contidos nos prontuários e das fichas de investigação das mortes maternas.

A pesquisa foi aprovada pelo Comitê de Ética em Pesquisa do Instituto Camillo Filho (CAAE 21164013.5.0000.5212). A confidencialidade das informações foi mantida, especialmente no que se refere ao sigilo dos nomes das mulheres presentes na DO, bem como os nomes dos profissionais envolvidos na assistência hospitalar e nos demais serviços de saúde contemplados pela investigação do óbito. Todas as entrevistadas assinaram termo de consentimento livre e esclarecido.

\section{Resultados}

\section{Mortes maternas de adolescentes}

Entre 2008 e 2013, foram registradas 290 mortes maternas no Piauí. Dessas, 50 (17,2\%) delas ocorreram entre adolescentes de 14 a 19 anos. A razão de mortalidade materna no estado variou de 113,9 mortes/100.000 nascidos vivos (2008) para 94,8 mortes/100.000 nascidos vivos (2013). A maior parte das mortes $(n=39 ; 78 \%)$ foi de adolescentes residentes em 34 cidades do interior do estado. O maior número de óbitos (64\%) ocorreu em Teresina (PI): uma concentração em decorrência do deslocamento de 21 (42\%) adolescentes de cidades cujas distâncias variaram de 52 a 746 $\mathrm{km}$ até a capital (figura 1). 
Figura 1. Mapa da distribuição das cidades com mortes maternas de adolescentes. Piauí, 2008-2013

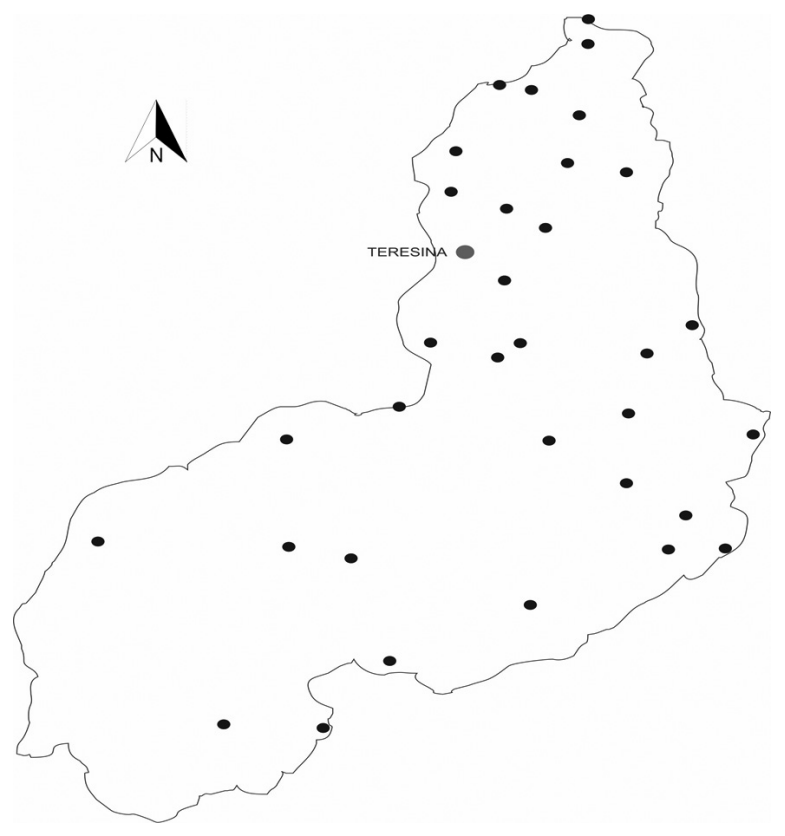

A tabela 1 evidencia que quase a metade $(n=24 ; 48 \%)$ das adolescentes com morte materna tinha entre 14 e 17 anos. A maior parte delas foi caracterizada, nas DOs, como sendo negras ( $\mathrm{n}=35 ; 70 \%)$, solteiras $(\mathrm{n}=18 ; 36 \%)$ e com 4 a 12 anos de estudo $(n=24 ; 48 \%)$. Houve maior prevalência de adolescentes na primeira gravidez ( $\mathrm{n}=27 ; 54 \%$ ) e com realização de mais de 06 consultas de pré-natal $(n=28 ; 56 \%)$. $10 \%(\mathrm{n}=5)$ das adolescentes não realizaram qualquer consulta pré-natal. A maioria das adolescentes morreu após o parto ( $\mathrm{n}=31 ; 62 \%)$, sendo a cesárea $(n=18 ; 45 \%)$ o principal tipo de parto realizado.

Tabela 1. Características das adolescentes com mortes maternas. Piauí, 2008-2013

\begin{tabular}{lrr}
\hline Variáveis & $\mathbf{n}$ & $\%$ \\
\hline Faixa etária (anos) & 24 & 48,0 \\
\hline 14 a 17 & 26 & 52,0 \\
18 e 19 & 6 & 12,0 \\
\hline Escolaridade (anos de estudo)1 & 11 & 22,0 \\
\hline 1 a 3 & 13 & 26,0 \\
4 a 7 & & 70,0 \\
8 a 12 & 35 & 14,0 \\
\hline Raça/ cor da pele ${ }^{2}$ & 7 & 7 \\
\hline Negra & 13 \\
Branca & & \\
\hline
\end{tabular}


Tabela 1. (cont.)

\begin{tabular}{|c|c|c|}
\hline Variáveis & $\mathrm{n}$ & $\%$ \\
\hline \multicolumn{3}{|l|}{ Estado civil ${ }^{3}$} \\
\hline Solteira & 18 & 36,0 \\
\hline Casada & 10 & 20,0 \\
\hline Outra & 2 & 4,0 \\
\hline \multicolumn{3}{|c|}{ Número de gestações anteriores ${ }^{4}$} \\
\hline Nenhuma & 27 & 54,0 \\
\hline 1 & 11 & 22,0 \\
\hline 2 ou mais & 2 & 4,0 \\
\hline \multicolumn{3}{|c|}{ Realização de pré-natal 5} \\
\hline $\operatorname{sim}$ & 28 & 56,0 \\
\hline Não & 5 & 10,0 \\
\hline \multicolumn{3}{|c|}{ Número de consultas de pré-natal } \\
\hline 1 a 5 & 11 & 39,3 \\
\hline \multicolumn{3}{|l|}{6 ou mais } \\
\hline \multicolumn{3}{|l|}{ Tipo de parto 6} \\
\hline Cesárea & 18 & 45,0 \\
\hline Vaginal & 13 & 32,5 \\
\hline Não se aplica & 9 & 22,5 \\
\hline \multicolumn{3}{|l|}{ Momento do óbito 7} \\
\hline Gravidez ou parto & 10 & 20,0 \\
\hline Após o parto & 31 & 62,0 \\
\hline Após o aborto & 5 & 10,0 \\
\hline
\end{tabular}

Fonte: Elaboração própria.

1Sem preenchimento na declaração de óbito: 20 casos (40,0\%); 2 Sem preenchimento na declaração de óbito: 8 casos (16,0\%); 3 Sem preenchimento na declaração de óbito: 20 casos (40,0\%); 4 Sem preenchimento na declaração de óbito: 10 casos (20,0\%); 5 Sem preenchimento na declaração de óbito: 17 casos (34,0\%); ${ }^{6}$ Sem preenchimento na declaração de óbito: 10 casos (20,0\%); ${ }^{7}$ Sem preenchimento na declaração de óbito: 4 casos $(8,0 \%)$.

Os transtornos hipertensivos (pré-eclâmpsia, eclâmpsia e/ou hipertensão arterial) foram as principais causas $(\mathrm{n}=14 ; 28 \%)$ declaradas de óbito entre as adolescentes. Logo após, foram registrados infecção puerperal $(n=8 ; 16 \%)$, hemorragias $(n=6 ; 12 \%)$, tromboembolismo $(n=6 ; 12 \%)$ e aborto $(n=5 ; 10 \%)$. Causas não obstétricas, como complicações anestésicas $(n=2 ; 4 \%)$, transtornos mentais $(n=2 ; 4 \%)$, transtornos do fígado $(n=1 ; 2 \%)$ e doenças infecto-parasitárias $(\mathrm{n}=2 ; 4 \%)$ também estiveram entre as causas básicas relatadas. Além disso, existiram 3 (6\%) mortes designadas na DO como não especificadas.

\section{Histórias de mortes de adolescentes por aborto}

O aborto foi declarado como causa de morte de seis adolescentes no período estudado, porém, dois casos foram excluídos. No primeiro, houve impossibilidade de localização do endereço declarado na DO. O óbito, ocorrido em 2012, teve atraso superior a dois anos na 
inserção dos dados no módulo digital do SIM. A ficha-síntese registrou a informação de que a adolescente teria usado remédio abortivo, sem outros detalhes. A DO, por sua vez, foi preenchida como 'morte obstétrica de causa desconhecida', todavia, a investigação não foi realizada como prevê o Ministério da Saúde para morte com causa mal-definidal8. No segundo caso, o óbito foi excluído porque, na fase de entrevista, descobriu-se que a idade informada na DO (12 anos) estava incorreta (era, na verdade, 24 anos), e, apesar da investigação, o erro não tinha sido corrigido. Tratava-se de morte por aborto, mas não de adolescente.

Oito entrevistas foram realizadas entre setembro de 2014 e junho de 2015 , sendo que em 02 casos houve necessidade de 03 entrevistas. As famílias residiam em Teresina e em cidades do interior, como Campo Maior, Luzilândia e União. Em todos os casos, o contato prévio com as famílias foi intermediado pela equipe da Saúde da Família local. As adolescentes residiam com os pais (03) e com a sogra (01). Todas as mães das adolescentes concordaram em participar do estudo, fornecendo, inclusive, registros de memórias das filhas, como fotografias e escritos.

\section{Primeira história}

Aos 16 anos e estudante do $3^{\circ}$ ano do ensino médio, a primeira adolescente residia com os pais e um casal de irmãos em área urbana de cidade próxima à capital. Sua mãe e irmã não souberam precisar em qual momento ela descobriu a gravidez, mas disseram que houve tentativas sem sucesso de aborto com medicamento pela adolescente, com uso persistente do remédio mesmo com o avanço da gravidez. Dose e forma de uso não eram conhecidas pela família. Para a família, a adolescente negou estar grávida até o desfecho do parto de feto morto.

Ainda grávida, passou por dois hospitais, com fortes dores abdominais e sempre acompanhada da mãe. A mãe a interrogava sobre a possibilidade de os sintomas serem decorrentes de possível gravidez, mas a adolescente mantinha a negativa. No primeiro hospital, em sua cidade, não foi examinada, mas recebeu medicamento com a justificativa de se tratar de cólica renal. Não tendo apresentado melhora no mesmo dia, foi encaminhada para o hospital geral, localizado na capital, a 120 quilômetros de distância. No segundo hospital, a gravidez avançada foi a hipótese principal, sendo a adolescente então encaminhada para a maternidade local.

$\mathrm{Na}$ admissão no último hospital, concluíram tratar-se de condição que complicava a gravidez: "morte fetal e infecção materna". A mãe da adolescente, lamentando o ocorrido, explicou que não tinha conhecimento do estado da filha, e informou que havia grande possibilidade de que a adolescente tivesse tomado medicamento para abortar, já que escondeu da família a gravidez. A mãe conta que perguntou ao médico sobre os riscos à saúde da filha, e a resposta teria sido a gravidade do estado, "pois ali havia uma situação de aborto provocado".

O feto foi natimorto, e a adolescente morreu por hemorragia e infecção generalizada dois dias após o parto. A causa básica da morte foi declarada como "aborto retido", e as informações existentes registram "comprometimento dos órgãos vitais por sepse”. Não há dados que expliquem os danos causados pelo método utilizado, porém, persistiu a alegação dos profissionais para a família, segundo os quais as mortes tanto da adolescente quanto do feto teriam sido causadas pelo aborto provocado. Também não existem explicações para o registro inadequado da causa básica da morte da adolescente - o "aborto retido".

\section{Segunda história}

A segunda história foi de uma adolescente de 18 anos, negra, estudante do $2^{\circ}$ ano do ensino médio, solteira, com uma filha de 3 anos. Morava com a mãe, três irmãs, um irmão e a filha, a $250 \mathrm{~km}$ da capital. Uma renda de aposentaria sustentava a família. O pai biológico da criança tinha outra família e não assumiu nenhuma responsabilidade sobre a 
filha. A mãe, com 60 anos, não conhecia o atual namorado e se preocupava com os "descuidos" da adolescente com relação à prevenção de gravidez, pois "já tinha falhado uma vez".

Dores fortes no baixo ventre se iniciaram e foram acompanhadas de sangramento vaginal dez dias antes de sua morte. Ela tinha percebido atraso menstrual, além de sintomas de gravidez, e havia usado medicamentos. Era tudo que se sabia. Os sintomas se exacerbaram e foram acompanhados de febre no dia da ida ao primeiro hospital, na cidade de residência. As dores fortes fizeram a adolescente e a mãe procurarem atendimento. $\mathrm{O}$ atendimento médico teria prescrito analgésico e encaminhado para o hospital da capital, com suspeita de apendicite.

No dia seguinte, já no hospital de referência, uma ultrassonografia comprovou a gravidez e a presença de batimentos cardíacos fetais. De acordo com a mãe, o profissional teria informado que seria necessária uma internação em maternidade para controlar o sangramento que estava aumentando. $\mathrm{O}$ diagnóstico dado à mãe da adolescente foi de ameaça de aborto. No serviço para o qual foi encaminhada, a adolescente ficou internada para tratamento clínico. A mãe, que a acompanhava, precisou retornar para casa e deixou como acompanhante uma tia.

No quarto dia, a adolescente se comunicou por telefone com a mãe, relatando que estava muito insatisfeita e incomodada com a internação, explicando que a dor permanecia e não sentia melhoras no quadro. A adolescente dizia que se sentia fraca, ainda com sangramento vaginal, mas a informação que recebia quando se queixava para algum profissional era de que deveria esperar, que a melhora iria ocorrer. No dia seguinte à comunicação com a mãe, a menina fugiu da maternidade, retornando para casa. As dores e o sangramento transvaginal aumentaram, com eliminação de "uma bola de sangue grande", na noite do mesmo dia, segundo a mãe.

A mãe a levou novamente ao hospital local. A adolescente apresentava febre e calafrios. Novamente, foi transferida para Teresina, onde passou mais três dias internada com infecção grave, em Unidade de Terapia Intensiva, onde morreu por "sepse". Durante essa segunda internação, foi submetida à cirurgia pélvica, com histerectomia. Os dados do prontuário registram "comprometimento de órgãos pélvicos por infecção do aparelho digestivo", sem especificação de qual órgão digestivo.

\section{Terceira história}

A terceira adolescente morava em Teresina. Ela tinha 18 anos, era empregada doméstica, casada, negra, com uma filha de 2 anos e 6 meses. Completou o ensino médio e vivia em união estável há 2 anos. Um ano antes, fez um aborto, no segundo mês de gravidez, com medicamentos. Não houve complicações, contudo, foi necessário fazer curetagem uterina. Segundo a sogra, com a descoberta de nova gravidez, através do beta-HCG, decidiu usar medicamentos outra vez.

A mãe relata que os quatro comprimidos do medicamento custaram duzentos e cinquenta reais, metade do seu salário. O medicamento foi tomado com mais de três meses do atraso menstrual. A adolescente tomou a mesma dose que usara na vez anterior. Ficou aguardando o início do sangramento vaginal por três ou quatro dias. Começou a sentir cólicas mais fortes do que o sangramento, o que a fez procurar o serviço médico de urgência, acompanhada da sogra e do parceiro. Pensava que já estaria na hora de fazer a curetagem, como fez anteriormente. Ao ser atendida, no entanto, recebeu uma prescrição de analgésico e a sugestão para procurar o pré-natal, porque se tratava "apenas de uma ameaça de aborto, com feto vivo e normalzinho, pela ultrassonografia", segundo a narrativa da sogra.

Uma semana se passou entre a consulta na urgência e o início de sangramento intenso e sintomas de febre, quando a adolescente retornou ao mesmo hospital. Os dados do prontuário informam que o diagnóstico da internação foi "aborto infectado". Entretanto, houve prescrição de medicamento para inibir o aborto. Dessa vez, a ultrassonografia visualizava uma gravidez de 14 semanas. A internação 
transcorria, a adolescente apresentava sangramento vaginal persistente, que lhe causou "anemia acentuada". A gravidez foi mantida, apesar do agravamento do quadro clínico. Segundo a mãe, a adolescente "não tinha forças para levantar". As explicações para as aflições da família eram de que a presença de viabilidade gestacional justificava a conduta expectante para o caso.

Duas semanas mais tarde, o quadro clínico da adolescente piorou, e foi recomendada a transferência para maternidade terciária. $\mathrm{O}$ diagnóstico na admissão do referido hospital foi de "choque séptico". Nova ultrassonografia foi realizada, cujo laudo evidenciou "morte fetal", e, nesse momento, houve indicação de curetagem uterina. Após o esvaziamento uterino, a adolescente foi encaminhada para a Unidade de Terapia Intensiva, onde permaneceu por um mês, sem melhora clínica. Dada a gravidade do caso, e a pedido da assistente social, a mãe se dirigiu àquele setor para autorizar a realização de traqueostomia. Além da informação sobre o estado grave da filha, recebeu informações sobre os gastos financeiros que o serviço dispensava durante toda a estadia no hospital. Após 45 dias de internação, a adolescente morreu por "sepse".

\section{Quarta história}

Aos 18 anos, solteira, negra, estudante do $1^{\circ}$ ano do ensino médio. A adolescente residia com a mãe e era a única filha. Estaria em seu primeiro namoro havia alguns meses, quando deu à mãe a notícia da gravidez e afirmou que desejava seguir com a gestação, dando início ao pré-natal. De acordo com a mãe, o namorado aceitava a situação. Após o terceiro mês, a mãe percebeu mudanças emocionais na adolescente, descrevendo-as como normais ao período. Mas também percebeu que ela se queixava de cólicas com muita frequência.

Em uma consulta de pré-natal, mãe e filha receberam orientação para fazer uma avaliação em ambulatório especializado, em decorrência de exame alterado para toxoplasmose. A mãe a acompanhou na consulta do serviço recomendado. Lá receberam receita para o tratamento, sem muitas explicações. O elevado custo dos remédios impossibilitou o tratamento. Semanas depois dessa consulta, a adolescente começou a apresentar sangramento vaginal abundante.

A mãe levou-a para a urgência da maternidade. Ao final do atendimento, o médico teria informado que havia óbito fetal. Perguntou sobre intercorrências no pré-natal. A mãe, então, relatou sobre a possível toxoplasmose e a não aderência ao tratamento, e, de acordo com sua narrativa, o médico concluiu que a provável causa da morte fetal seria a infecção. Diante disso, a adolescente deveria ficar internada para tratamento. A mãe não pôde acompanhar a filha, mas ficou esperando notícias em sua residência. Ao retornar à maternidade, uma pessoa comunicou que a adolescente não resistira e morrera por infecção. A explicação dada foi sobre o possível acometimento orgânico pela toxoplasmose.

Os registros não indicam em que medida a toxoplasmose acometeu a adolescente para causar dano que justificasse a falência de órgãos vitais. Não existe laudo de necropsia, apesar de o serviço existir na capital. Ao ser interrogada se a filha teria induzido o aborto, a mãe respondeu que não sabia, porque a adolescente não tinha lhe dito nada a esse respeito. Porém, uma amiga revelou que a adolescente fez uso de medicamentos por temer as graves complicações fetais após o diagnóstico da toxoplasmose. A mãe informou que, se a filha usou medicamento abortivo, foi porque tinha decidido não seguir com uma gravidez comprometida por uma doença que não tinha conseguido tratar. Não há registro em prontuário, nem relato da mãe sobre as consequências de ser portadora de toxoplasmose na gravidez.

\section{Discussão}

A morte de mulheres no início da vida reprodutiva por aborto é considerada uma morte evitável e que, por isso, viola direitos fundamentais. O presente estudo mostra que, no Piauí, $17,2 \%$ 
das mortes maternas foram de adolescentes, semelhante ao percentual da região Nordeste $(16,1 \%)$ e do País (14\%)18. Similar ao perfil encontrado em outras localidades ${ }^{16,17,19}$, elas eram adolescentes negras, de baixa escolaridade, em sua primeira gestação e residentes no interior do estado. Vale ressaltar, ainda, a frequência de não preenchimento dos campos que informam dados epidemiológicos, tanto na declaração de óbito quanto na ficha-síntese de investigação da morte, com destaque para o campo 'cor' (ausente em 16\%) e 'escolaridade' (ausente em 40\%). Esse fato evidencia a baixa qualidade de algumas das informações presentes no sistema de informação sobre mortalidade, com prejuízo para a auditoria adequada dos óbitos ${ }^{13}$.

As adolescentes residiam no interior, mas morreram na capital, Teresina, por ser o único serviço de assistência terciária à mulher no ciclo grávido-puerperal. Já foi demonstrado que quanto maior a distância entre serviços de saúde e mais longo o tempo no deslocamento, mais elevada a prevalência de mortes maternas pelo atraso no atendimento especializado $20,21$. Além disso, é possível que a situação social de vulnerabilidade (pobreza, baixa escolaridade, solidão, estigma) seja um fator adicional para o desfecho trágico.

No Brasil, a razão de mortalidade materna apresentou declínio de $56 \%$ nas últimas décadas, passando de 143,2/100 mil nascidos vivos em 1990 para 62/100 mil nascidos vivos em $2015^{22,23}$. Uma pesquisa que estimou a RMM entre 2008 e 2011 no Brasil identificou disparidades regionais, tendo a mais elevada taxa sido observada no estado do Maranhão (114,0/100 mil nascidos vivos), e a mais baixa em Santa Catarina (36,9/100 mil nascidos vivos). A metodologia de estimação levou em consideração causas comuns de mascaramento de mortes maternas, como o sub-registro de óbitos, a proporção de mortes de mulheres em idade fértil que foi investigada e a proporção de óbitos maternos não declarados na DO, e foram atribuídos a outras causas ${ }^{24}$. No presente estudo, a RMM do Piauí foi elevada e com valores superiores aos que deveriam ter sido alcançados pela $5^{\text {a }}$ Meta de Desenvolvimento ${ }^{25}$, sugerindo a ausência de políticas para melhorar o indicador de saúde materna no período analisado.

As causas das mortes das adolescentes deste estudo foram semelhantes às de mulheres de outros grupos etários e outras regiões do Brasil e do mundo 26,27. Apesar de amplas variações regionais, hemorragias, transtornos hipertensivos, infecções e complicações do aborto inseguro contribuem para mais da metade dos óbitos obstétricos diretos em países em desenvolvimento. Por sua vez, as causas indiretas, que ainda predominam na África subsaariana, são responsáveis por cerca de um quarto das mortes ${ }^{26}$. Considerados evitáveis em sua grande maioria, 78\% (n=39) dos óbitos maternos de adolescentes neste estudo foram determinados por causas obstétricas diretas. É consenso que o emprego adequado de antibióticos, o uso sistemático de ocitocina pós-parto e a utilização oportuna do sulfato de magnésio são medidas eficazes e seguras para a redução da mortalidade materna ${ }^{\mathbf{2 2 , 2 6}}$.

Acometendo mulheres de todas as idades no período reprodutivo, o aborto contribui de forma significativa para a elevação da RMM, uma vez que está entre a terceira e a quarta causa de morte materna nos países em desenvolvimento ${ }^{\mathbf{2 6} 2 \mathbf{2 8}}$. Mais prevalente em locais onde há legislação proibitiva ao aborto legal, o óbito por aborto geralmente está associado ao retardo no atendimento ou no diagnóstico de complicações ${ }^{10,12,14}$. No entanto, a criminalização de sua prática não impede que as mulheres interrompam a gravidez de maneira insegura, amplificando apenas o risco de desfecho negativo, ${ }^{\mathbf{9}}$. Um estudo caso-controle realizado em Teresina (2012-2013) para avaliar morbidade materna grave/near miss demonstrou que o aborto, resultando em infecção e hemorragia, foi a principal causa isolada de morte materna, sendo responsável por 3 entre 10 mortes identificadas no período 29.

Esta pesquisa identificou cinco adolescentes mortas por aborto, com muitos pontos 
de intersecção nas narrativas apresentadas. Primeiro, houve utilização de medicamentos, adquiridos no mercado clandestino. Segundo, todas procuraram o serviço de saúde em algum momento das suas trajetórias, porém, esse atendimento não impediu suas mortes. Por fim, foi comum a demora na busca do serviço de saúde para atendimento, seja pelo estigma, por medo de denúncia à polícia ou por receio de discriminação nos serviços de atendimento. Não menos importante, também, foi o retardo dos profissionais dos serviços em diagnosticar e tratar as complicações que levaram a adolescente à morte.

Esse retardo no diagnóstico e/ou tratamento das complicações do aborto, já observado em outros cenários ${ }^{31}$, ficou evidente em todos os relatos. Nesse sentido, merece destaque a terceira história, da adolescente que morreu no $45^{\circ}$ dia de internação, cujo diagnóstico dado no momento da admissão foi de aborto infectado, com 14 semanas de gestação. $\mathrm{O}$ exame ultrassonográfico inicial havia demonstrado feto vivo, porém, com redução acentuada de líquido amniótico. A despeito da piora gradativa da adolescente, a interrupção da gravidez só foi realizada por curetagem uterina, 15 dias após a internação, quando novo exame ultrassonográfico mostrou feto morto, e já havia o quadro de sepse instalado. Se a recomendação da norma técnica do Ministério da Saúde tivesse sido considerada $^{\mathbf{3 0}}$, o esvaziamento uterino (independente das condições fetais) deveria ter ocorrido logo após a admissão da adolescente no hospital terciário, tendo em vista o elevado risco de agravamento de sua saúde e a grande possibilidade de morte.

Este estudo apresenta algumas limitações que merecem ser consideradas, principalmente relacionadas com a qualidade de registro das mortes. Primeiro, chama a atenção a ausência de dados importantes em diversas declarações de óbito, tais como causa de morte não codificada nos capítulos da CID10 referentes a mortes maternas, bem como o não preenchimento dos campos 'cor', 'situação conjugal' e 'escolaridade'.
Segundo, também havia escassez de informações nas fichas-síntese, instrumentos importantes na investigação dos óbitos e que poderiam dirimir dúvidas ou corrigir erros existentes na DO. Terceiro, os dados obtidos das entrevistas podem ter sido mascarados tanto pelo viés de memória como pelo estigma do aborto entre os familiares. Apesar dessas limitações, este estudo lança novas luzes sobre a evitabilidade das mortes maternas de adolescentes. Se, por um lado, o perfil do óbito é semelhante ao das mulheres adultas, por outro, há evidência de amplificação da vulnerabilidade própria da adolescência, principalmente nas mortes decorrentes do aborto.

\section{Considerações finais}

Há carência de estudos sobre mortalidade por aborto no Brasil, porém, a urgência de dados confiáveis se impõe no momento em que se discute a descriminalização do aborto até 12 semanas na Suprema Corte. O perfil dos óbitos maternos de adolescentes encontrado é comum em localidades ainda com vulnerabilidade econômica e social, como o estado do Piauí. O principal resultado deste estudo, destacado pelas histórias das adolescentes, é que as mortes poderiam ter sido evitadas se tivesse ocorrido maior agilidade na assistência. A demora em procurar os serviços de saúde, o silêncio em torno dos métodos de aborto utilizados, a dificuldade em investigar as queixas e o obstáculo em tratar as complicações culminaram com a oportunidade perdida no cuidado das adolescentes em suas necessidades de saúde. Imersas em ambientes de desigualdades, as adolescentes deste estudo provavelmente enfrentaram variadas barreiras para o livre exercício de seu planejamento reprodutivo. Tendo em vista que o acesso à interrupção segura da gestação é um direito reprodutivo ${ }^{32}$, múltiplas ações deveriam ser implementadas para reduzir desfechos negativos e possibilitar 
às adolescentes oportunidade de assumir o controle sobre sua saúde sexual e reprodutiva. Se o acesso pleno à saúde reprodutiva for considerado como passo necessário para a redução da mortalidade materna, tópicos como educação sobre sexualidade, oferta de métodos contraceptivos eficazes e redução dos abortos inseguros devem ser prioritários nessa agenda. Quando o tema do aborto for encarado como política de saúde no País, o imperativo deverá ser a proteção das mulheres e não sua criminalização.

\section{Colaboradores}

Nunes MDS (0000-0002-6209-3025)*, Madeiro A (0000-0002-5258-5982)* e Diniz D (0000-0001-6987-2569)* contribuíram igualmente na elaboração do manuscrito.

\section{Referências}

1. Neal S, Matthews Z, Frost M, et al. Childbearing in adolescents aged 12-15 years in low resource countries: a neglected issue. New estimates from demographic and household surveys in 42 countries. Acta Obstet Gynecol Scand 2012; 91(9):1114-8.

2. Brasil. Ministério da Saúde, Secretaria de Vigilância em Saúde. Saúde Brasil 2014: uma análise da situação de saúde e das causas externas. Brasília, DF: Ministério da Saúde, 2015.

3. Vaz RF, Monteiro DLM, Rodrigues NCP. Trends of teenage pregnancy in Brazil, 2000-2011. Rev Assoc Med Bras. 2016; 62(4):330-5.

4. Amorim MMR, Lima LA, Lopes CV, et al. Fatores de risco para a gravidez na adolescência em uma maternidade-escola da Paraíba: estudo caso-controle. Rev. Bras. Ginecol. Obstet. 2009; 31(8):404-10. and Contributor ID).
5. Instituto Brasileiro de Geografia e Estatística. Esta- tísticas do registro civil, v. 41. 2014. Rio de Janeiro: IBGE, 2015.

6. Berquó E, Garcia S, Lima L. Reprodução na juventude: perfis sociodemográficos, comportamentais e reprodutivos na PNDS 2006. Rev. Saúde Pública. 2012; 46(4):685-93.

7. Nunes MD, Madeiro A, Diniz D. Histórias de aborto provocado entre adolescentes em Teresina, Piauí, Brasil. Ciênc. Saúde Colet. 2013; 18(8):2311-8.

8. Menezes GMS, Aquino EML, Silva DO. Aborto provocado na juventude: desigualdades sociais no desfecho da primeira gravidez. Cad. Saúde Pública. 2006; 22(7):1431-46

9. Sedgh G, Singh S, Shah IH, et al. Induced abortion: incidence and trends worldwide from 1995 to 2008. The Lancet. 2012; 379(9816):625-32. 
10. Grimes DA, Benson J, Singh S, et al. Unsafe abortion: the preventable pandemic. The Lancet. 2006; 368(9550):1908-19.

11. Diniz D, Medeiros M, Madeiro A. Pesquisa Nacional de Aborto 2016. Ciênc. Saúde Colet. 2017; 22(2):65360 .

12. Calver C, Owolabi OO, Yeung F, et al. The magnitude and severity of abortion-related morbidity in setting with limited access to abortion services: a systematic review and meta-regression. BMJ Glob Health. 2018; 3:e000692.

13. World Health Organization. Global health estimates 2015: deaths by cause, age, sex, by country and by region, 2000-2015. Geneva: WHO, 2016.

14. Martins EF, Almeida PFB, Paixão CO, et al. Causas múltiplas de mortalidade materna relacionada ao aborto no estado de Minas Gerais, Brasil, 2000-2011. Cad. Saúde Pública. 2017; 33(1):e00133115.

15. Lima BGC. Mortalidade por causas relacionadas ao aborto no Brasil: declínio e desigualdades espaciais. Rev. Panam. Saúde Pública. 2000; 7(3):168-72.

16. Neal S, Mahendra S, Bose K, et al. The causes of maternal mortality in adolescents in low and middle income countries: a systematic review of the literature. BMC Pregnancy Childbirth. 2016; 16(1):352.

17. Ganchimeg T, Ota E, Morisaki N, et al. On behalf of the WHO Multicountry Survey on Maternal Newborn Health Research Network. Pregnancy and childbirth outcomes among adolescent mothers: a World Health Organization Multicountry Study. BJOG. 2014; 121(supl1):40-8.

18. Brasil. Ministério da Saúde. Saúde Brasil 2013: uma análise da situação de saúde e das doenças transmissíveis relacionadas à pobreza. Brasília, DF: Ministério da Saúde; 2014.

19. Leal MC, Gama SGN, Cunha CB. Desigualdades raciais, sociodemográficas e na assistência ao pré-na- tal e ao parto, 1999-2001. Rev. Saúde Pública. 2005; 39(1):100-7.

20. Hernandez JC, Moser CM. Community level risk factors for maternal mortality in Madagascar. African J Reprod Health. 2013; 17(4):118-29.

21. Chen YN, Schimitz MM, Serbanescu F, et al. Geographic access modeling of emergency obstetric and neonatal care in Kigoma Region, Tanzania: transportation schemes and programmatic implications. Glob Health Sci Pract. 2017; 5(3):430-45.

22. Alkema L, Chou D, Hogan D, et al. Global, regional and national levels and trends in maternal mortality between 1990 and 2015, with scenario-based projections to 2030: a systematic analysis by the UN Maternal Mortality estimation inter-agency group. The Lancet. 2016; 387(10017):462-474.

23. Brasil. Ministério da Saúde [internet]. DATASUS. Estatísticas Vitais. Mortalidade - 1996 a 2016. Óbitos de mulheres em idade fértil e óbitos maternos [acesso em 2018 jul 30]. Disponível em: http://www2.datasus.gov.br/DATASUS/index.php?area $=0205 \& i d=6$ 939\&VObj=http://tabnet.datasus.gov.br/cgi/deftohtm.exe?sim/cnv/matio.

24. Szwarcwald CL, Escalante JJC, Rabello Neto DL, et al. Estimação da razão de mortalidade maternal no Brasil, 2008-2011. Cad. Saúde Pública 2014; 30(supl):7183.

25. United Nations. The Millennium Development Goals Report 2014 [internet]. New York: United Nations; 2014. [acesso em 2018 ago 7]. Disponível em: http:// www.un.org/millenniumgoals/2014\%20MDG\%20 report/MDG\%202014\%20English\%20web.pdf.

26. Say L, Chou D, Gemmili A, et al. Global causes of maternal death: a World Health Organization systematic analysis. The Lancet. 2014; 2(6):323-33.

27. Silva BG, Lima NP, Silva SG, et al. Mortalidade materna no Brasil de 2001 a 2012: tendência temporal e diferenças regionais. Rev. Bras. Epidemiol. 2016 
;19(3):484-93.

28. Kassebaum NJ, Bertozzi-Villa A, Coggeshall MS, et al. Global, regional, and national levels and causes of maternal mortality during 1990-2013: a systematic analysis for the Global Burden of Disease Study 2013. The Lancet. 2014; 384(9947):980-1004.

29. Madeiro AP, Rufino AC, Lacerda EZG, et al. Incidence and determinants of severe maternal morbidity: a transversal study in a referral hospital in Teresina, Piauí, Brazil. BMC Pregnancy Childbirth. 2015; 15:210.

30. Brasil. Ministério da Saúde, Secretaria de Atenção à Saúde, Departamento de Ações Programáticas Estra- tégicas. Atenção humanizada ao abortamento: norma técnica. Brasília, DF: Ministério da Saúde, 2011.

31. Araújo TVB, Aquino EML, Menezes GMS, et al. Delays in access to care for abortion-related complications: the experience of women in Northeast Brazil. Cad. Saúde Pública. 2018; 34(6):e00168116.

32. Cook RJ, Dickens BM. From reproductive choice to reproductive justice. Int J Gynaecol Obstet. 2009; 106(2):106-9.

Recebido em 06/03/2019

Aprovado em 13/09/2019

Conflito de interesses: inexistente

Suporte financeiro: não houve 